tion to en arroncous ahemical theors in trentment; hence, inconsintent as it mas appear, I should have no hesitation, undor certain circumstances, to emplos soids in the trentment of a dyspeptic pationt, thongh troubled with various exmptoms of acidity; or to use castor oil, or cod-liver oil, or oily emulsions, or to allow a moderate quantity of $80 \mathrm{mo}$ fetty aliments where they might seem to be called for, slthough I might have reason to suspect fatty degeneration in some particular organ.

Although in diabetes I attach the primary importance to the digestive organs, and more especially to the stomach, liver, and pancreas, and to some undetermined condition of the nervous system by which these organs are influenced, I am very far from limiting the interest to these organs. The kidneys also claim attention; and great importance is doubtless to be attached to the peculiar cachectic condition superinduced, very probably through the kidneys, by the disense.

I am induced to differ from Dr. West, and others who may regard the kidneys as only passively concerned in the - disease, by the following reasons:-In three or four instances I have met with diabetic patients, in which the disease occurred in families in which Dr. Bright's disease of the kidneys also occurred; and, in two of these examples, the family predisposition to this latter affection of the kidneys was very strongly marked. Again, the disturbance of the function of the kidneys does not seem to be limited to the mere passing off of the sugar brought to the kidneys by the blood; since, though urea is found in diabetic urine, it appears to be far below the healthy proportion. There is snother symptom which, though not immediately connected with the kidneys, will strengthen the presumption that they are materially concerned. The great diminution or total abolition of the sexual power, so intimately associated with this disease, and so characteristic of it, that a patient has told me that it was the first symptom which called his attention to his malady, cannot be ascribed to mere general debility; seeing that the symptom occurs when the patient, as respects the general powers of the system, is very much stronger than many advanced phthisical patients, and other extremely debilitated subjects, whose virility remains perfect. I conceive, therefore, that there must be some sort of reflex action upon the organs of generation, of which the starting point is in the kidneys. The idea does not appear to be an overstrained one, seeing that the renal and spermatic plexuses are so intimately connected with the same portion of the spinal cord.

Again, it seems scarcely possible that the kidneys could be subjected with perfect impunity to the constant passage of their secretion, inordinate in its quantity, and vitiated and morbid in its properties. Finally, the resulting cachexia is precisely such as is to be expected from the imperfect performance of the depuration of the blood by the kidneys, and should direct our attention to them, just as a livid countenance prompts us to look for derangement in the organs of respiration.

I would in the next place invite attention to the state of the tissues of the body in general, which constitutes the cachectic condition to which I have alluded. It seems to result from the combined action of the diffusion of the sugared blood over the system, and the imperfect elimination of those excrementitious matters which the kidneys are destined to throw off. I have already compared it to the effect produced by the employment of diseased rye. It ought also to be compared and contrasted with the cachexis resulting from Bright's disease of the kidneys, to which it must be considered as allied; and, to do the subject ample justice, it should be considered in conjunction with some other forms of cachexis which merit careful attention, and which require to be studied as the complement to the morbid anatomy of all the organs considered individually. To attempt to do this, even in the most cursory manner, would carry mo beyond the limits which ean be allowed to this paper.

8s, Bederd squere.

\section{CASE OF INTESTINAL OBSTRUCTION, 1 SUCCESSFULLY TREATED BY STRONG PURGATIVES.}

By J. G. DAVEY. M.D.

[Read at the Quarterly Meeting of the Bath and Bristol Branch, 28th September, 1854.j

Wrrzorr intending or even wishing to do else than arand all honour to the labours and successes of the operating surgeon, I would take the present opportunity of putting on record a case of intestinal obstruction, which, in $\mathrm{mJ}$ opinion, presents certain features of much interest, and of grest practical importance. At the present day, when 80 much is rentured on with the knife; when gastrotomy, ovariotomy, etc., have become mere matters of course; when the abdomen is fearlessly cut into, and its contents, with those of the pelvic cavity, examined with the same sang froid that characterises any unimportant surgical manipulation-it is well that we should pause in our high and ambitious career, and ask ourselves, whether too much has not been attempted; whether we are altogether justified either in exposing the vital abdominal organs to our view, and this when in doubt of the presence or otherwise of an incarcerated bowel; or in cutting away a tumour, which, if malignant, had better remain where it is; and which, if not malignant, admits of a successful and palliative treatment.

If I mistake not, the annexed case will be seen to justify the preceding brief though important reflections.

Case. On the evening of the 6th of June last, I was requested by Mr. Salmun, of Thornbury, to see with him R. W., aged 72 jears, a farmer, of industrious and temperate habits, and generally of sound health. On arriving at the house, we found our patient lying in bed on his back, with the knees drawn upwards towards the abdomen. He appeared much distressed; the countenance was anxious; and this and his general manner pourtrayed very urgent bodily uneasiness and mental inquietude. The skin was for the most part lax and warm, but in parts damp and even chilly. The pulse was about 90, full, but soft, and most easily compressible. Respiration was not much disturbed. On examination, the abdomen presented a well marked irregularity on its surface, as if the intestines wero unequally and disproportionately distended, as was really the case. There was present a very general uneasiness of the whole abdomen; but on its left side, close to the internal ring, he complained of pain, on even slight pressure. On inquiry, I learnt that $R$. W. had been the subject, for many years, of a reducible scrotal hernia; but that he had on no occasion, either remote or recent, experienced anj kind of inconvenience from this circumstance. My forefinger passed most readily into the external ring, and upwards through the inguinal canal itself, so relaxed and enlarged were the several parts entering into its formation. He complained of constant sickness; the matter ejected by the stomach being decidedly stercoraceous. The bowels had not been relieved, in the least degree, for the past six days.

Mr. Salmon informed me, in reference to the previous history of the case, that he visited $R$. W. on the and June, and found him complaining of much pain and uneasiness about the abdomen, with nausea and occasional slight sickness, and inaction of the bowels. Mr. Salmon ordered him a mixture of sulphate of magnesia and jalap; this producing no effect, some pills composed of gamboge, compound extract of colocynth, and scammony, were advised. Afterwards (on the 3nd), these failing to afford relief to the bowels, a large dose of blue pill and compound extract of colocynth was given, and followed by repeated doses of sulphate of magnesia. Bnall quantities of croton oil and calomel were given at the same time, to three doses. The symptoms still remaining urgent, on the morning of June 4th an enema of turpentine and castor oil, to which two drops of croton oil were added, wes administered; and the pills abore mentioned were oxdered to be continued. 
June 6th. He was still no better. A repetition of the pills and enems, as on the 4th, was ordered. It was on this day that the matter vomited presented a stercoraceous character. On the 6th, I saw the patient with Mr. Salmon. The condition in which I found $R$. W. is given above.

Having, as I thought, duly examined our patient, and made out all the facts of his case, it occurred to me that a small knuckle of intestine might have become strangulated near the upper or internal ring; that what had hitherto been a reducible scrotal hernia, had become in part, and superiorly, irreducible, and impacted in the part just mentioned; and if not so, that some portion or the other of the intestinal tube had become strictured. The pain situated at the internal ring looked, in itself, suspicious; but, inasmuch as this was as palpably, or even more so, to be referred to the distended and descending colon, contiguous to and beneath it, I felt my diagnosis in some difficulty. Reflecting on the case, in all its bearings, and on the treatment adopted by Mr. Salmon up to the period of my own visit, I felt there was but one thing left for me to do; viz., to give him the chance of drugs yet more potent than anything hitherto advised for his relief: and, inasmuch as those already given comprised the most active that we are in the habit of employing, I could only recommend the same in larger and more frequently repeated doses than Mr. Salmon had ventured on. In fact, I felt that the time had arrived when all ordinary modes of administering remedies would be of no avail, and were therefore to be discarded; and, in spite of the cautious directions of certain writers on materia medica and therapeutics as to the administration of medical substances to aged people, I determined on giving our patient, with the concurrence of Mr. Salmon, the benefit or otherwise of large doses. We administered, without loss of time, a bolus containing ten grains of calomel and the same quantity of the compound extract of colocynth, with the addition of two drops of croton oil. A mixture, containing aromatic spirits of ammonia and compound tincture of cardamoms in camphor mixture, was directed to be taken frequently, as a cordial or stimulant. Brandy and water was also prescribed, but in very small quantities, and from time to time. We hoped that, by allowing the stomach to remain pretty nearly empty, the bolus would be the more likely to be retained: nor were we disappointed. As it produced no effect on the bowels after four hours, a second, precisely similar to the first, was given.

June 7 th. At ten o'clock in the morning-i.e., on the morrow succeeding the evening on which I saw R. W.-the bowels were for the first time since his illness relieved; and by the afternoon of the same day, our patient had had as many as five copious evacuations. The sickness and pain passed away; in point of fact, $R$. W. was convalescent; and in two or three days he resumed his ordinary avocations.

Remakks. Now, the foregoing case becomes more particularly interesting when we compare it with one of much the same kind reported by Dr. Joseph Ridge, and read before the Hunterian Society in January of the present year. It is to be found at page 406 of the current volume of the Asgociation JournaL. Dr. Ridge's patient, a lad aged 14, was the subject of occasional attacks of abdominal uneasiness, with sickness and constipation; these had yielded to appropriate treatment up to the accession of the severeattack which terminated fatally in August of last year, viz., 1853. At this time, the bowels became again "inactive"; and, after some days, severe pain came on within the abdomen, and this was attended with vomiting. The countenance, Dr. Ridge tells us, was anxious, and the cheeks were flushed. A dose of castor oil was given, but rejected; and, after waiting some hours, the vomiting ceased; then two drachms of sulphate of magnesia, in dill-water, were administered, but this also "was cast up". E. N. became very much worse. Hot fomentations were applied to the abdomen. During the two following days, six grains of calomel were given in divided doses ; and four enemsta were administered, three of which were composed of castor oil and gruel, and one of castor oil, gruel, and black draught. These relieved the lower bowels of "most offensive matter"; but the patient was scarcely benefited by the treatment, inasmuch as the pulse was "weak" and "intermittent", and he became so delirious and excited as to require the efforts of the attendants to prevent his getting out of bed, and rushing about the room. From Tuesday evening he got worse and worse, and, in spite of the employment of morphia, prussic acid, efferrescing mixture with tincture of hyoscyamus, pills of compound extract of colocynth and extract of hyoscyamus, and draughts of the carbonate with small quantities of the sulphate of magnesia, etc., and in spite also of the performance of gastrotomy by Mr. Hilton, who found a portion of the jejunum in "an abnormal hole in the mesentery", the poor lad died.*

It appears, then, that up to a certain point these two cases cited were pretty nearly alike; $i$.e., they so far agreed in all essential characteristics. In the former case, the employment of additional and more active means of treatment, as suggested by myself in consultation with Mr. Salmon on the 6th of June last, resulted in the relief and restoration of the patient; whilst in the latter case, the performance of gastrotiomy was followed by the death of the patient. Under the circumstances, then, one is naturally led to inquire, in reference to E. N.: lst, Was the preliminary treatment of a sufficiently active character? Again, did all the facts of this case demand the performance of so fearful an operative procedure as that advised and practised? According tu the account published, E. N. had been "always liable to attacks of sickness and constipation", and "abdominal distress"; but these same attacks had hitherto yielded to appropriate treatment, " complete abstinence from food, and rest"; or, in other words, $\mathbf{E}$. N. had been always the subject of "partial descents of intestine through an opening which was found in the mesentery", and of "spontaneous reductions therefrom, promoted by recumbency", and by "the diminution of fulness of the stomach and bowels, and of the vessels of the structures concerned, and other influences". Such were the results obtained, on former occasions, from the treatment practised for his relief. It certainly does appear to me that the treatment of the last, the fatal attack, was not " of a sufficiently active character"; and hence was it, in my judgment, that no "spontaneous reduction" of the descended intestine, "through an opening which was found in the mesentery", took place. It will, I think, occur to the minds of several gentlemen whom I have the privilege to address, that, if called on to treat a case "of intestinal obstruction within the abdomen", to quote Dr. Ridge's words, they would hardly content themselves by ordering a dose of castor oil, and afterwards two drachms of sulphate of magnesia; and, on a subsequent day, a few grains of calomel, and then of a simple enema or two, effervescing mixture, and such like; and this after several days of obstinate inaction of the bowels, attended with alarming constitutional symptoms. And finding such means fail, how few practitioners, I should have thought, would have ventured either to advise or practise gastrotomy. I should mention here, that the medical treatment of this poor boy devolved not only on Dr. Ridge, but on two or three other gentlemen. Dr. Ridge was not consulted until the sixth day of the attack.

With regard to the operation itself. "The diagnostic signs of strangulation of any portion of the intestines were not considerable", writes Dr. Ridge. The abuomen was depressed at its lower half; there was a slight prominence just above and to the left of the umbilicus, where much tenderness existed. The upper part of the abdominal cavity was more tympanitic than the lower. However, the diagnosis arrived at by the gentlemen engaged with this case was of such a nature as to suggest to each one of them the necessity of gastrotomy; and Mr. Hilton's assistance was solicited, he having had some peculiar experience of such affections.

I would conclude by reminding gentlemen favourable to

Was not the relative position of a "portion of the jejunum" and the "mesontory" congenital? J. G. D. 


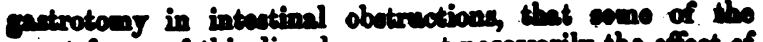

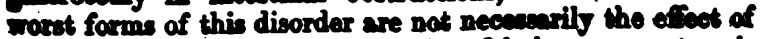
internal strangulation, so to spenk. Of three cases termimating fatelly in my own practice, in times gome by, on was the mere sccompaniment of scute inflammatory aotion involving the substance and peritoneal suriace of the nterus, the parts adjacent, and particularly the parietes of the descending colon. This was followed by adhesions of the fundus and peritoneel surface of the uterus to the walls of the large intestine lying in contact with each other, and $s 0$ was formed an opening between these viccors; and, as may be expected, the froces eventually passed per vagiratim. Fature here did what the knife could never acoomplinh. A second case of intestinal obstruction was caused by scute ovaritis. On examination after death, I found lying deeply pressed into the side of the rectum, placed in the hollow of the sacrum, and between the gut and the body of the nterus, occupying a large portion of the polvis itself, an indurated and large ovary, an incision into which wes followed by the escape of a quantity of thick purulent matter. The intestinal canal was obliterated by the great pressure of the inflamed and enlarged organ on the rectum, the anterior and posterior regions of which were in the closest contact. A puncture through the rectum might have saved the life of my patient. The third case of this kind occurring to myself was referrible only to a portion of the bowels becoming incarcerated. I found, on examination of this fatal case, a portion of dense fascia passing from the brim of the pelvis on the right side to the upper part of the sacrum, near the mesian line, leaving a space behind it. Between the posterior border of this same portion of fascia and the anterior ridge or line of the pelvis, i. e., in the spece so formed, was found a considerable portion of the small intestines; and this, becoming 80 distended as to lose its peristaltic action, caused the death of my patient, an old woman, demented and paralytic.

The foregoing facts command us to remember, then, that two out of three patients suffering from internal and intestinal obstruction may be without the reach of the surgeon's knife, as well as beyond the skill and appliances of the physician; and that, however much the one case in three may require operative assistance, this must be ro sorted to only when decided and actire means of treatmont, both general and medicinal, shall have failed to restore the parts affected by disorder, and never otherwise.

I would ask, in conclusion, What would have been, in all probability, the consequence of gastrotomy on Mr. Salmon's patient-what the effects of the non-employment of those active measures which resulted in his restoration? Again, what may have been the effects of active treatment in the case of E. N. - what the consequences to this poor boy, if he, at the same time, had not been the subject of a severe and formidable operation, though doubtless performed with the usual skill and dexterity of so good a surgeon as Mr. Hilton is known to be? I beg Dr. Ridge's pardon, if my remarts should seem discourteous; but the subject is one of some importance, and has therefore high claims on our consideration.

Northroods, near Briatol, Oetober 1854.

\section{COMPLETE CONSTIPATION OF THE BOWELS FOR TWENTY-EIGHT DAYS, TERMINATING FATALLY AFTER OPERATION.}

By GEORGE NORMAN, Esq., Surgeon to the Bath United Hospital.

[Read at the Quarterly Meeting of the Bath and Bristol Branch, September 28th, 1854.]

Try rubject of this case was a lady, aged 31, who had onfoyed good bealth in the early part of her life, who had been married six years, had never been pregnant, had menctruated very imperfoctly after her marriage, and that only for a short period, and in whom there had been for the last beur yeare no menetrwal discharge, excopting the occacional

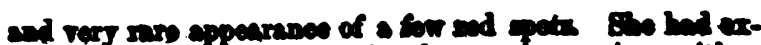

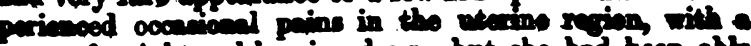
wanes of weight and bearing down; bet tho had been she to ride on horweback, and to wall moderate dintancea.

About two jears aince, aho was under treatment in Inomdon for uterine complaints. The body of the uterus was stated to be enlarged; the os utari contracted, and tender to the touch. Leeches were applied, and means were cm ployed to produce a ralaration of the $c$; and, after a time, nitrate of silver was introduced into the nterus, with the view of producing bloody discharge, in the expectation that the menstrual diecharge might be reproduced. This, horever; did not occur; and she returned home, and afterwand emplojed no other treatment for the uterine affection than the occasionsl use of saturnine injections per vaginaen. She continued to ride on horseback, to $8^{\circ}$ in a carriage, and to take some walking exercise.

When twelve jears old, she had a severe attack of constipation of the bowels; and from that time her bowels were more or leas costive, and she was obliged to take aperients somewhat frequently.

About three months after her return from London, she began to experience greater difficulty in procuring the evacuation of the bowels, and it was only by the use of injections that she had been able to effect the object; and latterly the difficulty had so increased that sho was obliged to use the injection three or four times a day, a small portion only of freces passing with each injection. She mont frequently administered the enems herself; and she had learnt to facilitate its action by pressing on the anus and perinæum after the warm water had been thrown up, which seemed to force the fluid higher up.

On Wednesday, the l0th of $\mathrm{May}$, she had an evacuation from the bowels without an enema-a very unusual circumstance. She was quite well, and went in a pony phaeton four or five miles to dine, returning in the evening. On the following day, she was seired with severe pain in the lower part of the abdomen, and in the pelvic region. On the next day, I believe, she sent for her usual medical attendant (Mr. William Walker, of Shepton Mallet), who found considerable pain and tenderness over the abdomen, intolerance of pressure, and other symptoms indicating to him the existence of peritonitis, and it might be also of enteritis. He bled her at the arm, which somewhat relieved the pain; he also gave mild aperients, which, however, produced no effect. The next day, the pain and tenderness continuing, he applied leoches to the abdomen, and continued to give mild aperients and enemats, without producing any frecal evacuation. Another medical gentleman (Mr. Flower, of Chilcompton) was called to the assistance of the former attendant; and he endearoured to pass a long elastic tube. He thought that, when passing his finger high up, he had felt an open space, into which he passed the tube, and then threw up warm water, which returned without producing any eracuation of fæces. Calomel and opium were given every four hours; and subsequently. croton oil was given, without any beneficial effect, and, after a time, it produced romiting, which continned as often as anything was taken into the stomach, but without any frocal smell or appearance.

May 15th. I sam this lady with the two gentlemen who had attended her. The romiting had somewhat subeided, but had not entirely ceased : there was no freculent appearance in what had been thrown up. Bhe had not a great deal of pain ; her pulse was quick, but firm; and bet countenance was tolerably good. There was but little pain on preseure on any part of the abdomen, but very considerable distension. The whole surface of the abdomen was resonant, excepting the left iliac region. The finger pessed into the rectum found the gut large and empty; but I could not find any aperture leading out of it. The fundus of the uterus, felt through the rectum, was large and irregular; and is appeared to occupy a considerable portion of the pelvie Attempts were made to pace bougies of dir aruats finds higher up than the finger could remch, but withonts enccess. It was decided that no purgatives should it 